\title{
Systematic bottom-up theory for the viscoelastic response of worm-like-chain networks
}

\author{
Tetsuya Hiraiwa ${ }^{1,2}$ and Roland R. Netz ${ }^{1}$ \\ 1 Fachbereich Physik, Freie Universität Berlin, Arnimallee 14, 14195 Berlin, Germany \\ 2 Department of Physics, The University of Tokyo, Hongo, Tokyo 113-0033, Japan
}

\author{
PACS 87.16.Ln - Cytoskeleton \\ PACS 83.60.Bc - Linear viscoelasticity \\ PACS 87.16.A- - Theory, modeling, and simulations
}

\begin{abstract}
Using the frequency-dependent force-extension response functions of single worm-like chains as the only input, the linear complex viscoelastic modulus $G(\omega)$ of a polymeric network with given connectivity is derived from a systematic bottom-up theory via iterative coarse-graining. Choosing a cubic connectivity and accounting for random network orientation, we find excellent agreement with experimental data for actin networks under shear over the entire frequency range with the strength of the osmotic pressure that acts within the polymeric network as the only fitting parameter. In particular, we obtain a viscoelastic plateau regime at low frequencies and a crossover to an intermediate frequency regime characterized by a power-law behavior $G(\omega) \propto \omega^{1 / 2}$ and an inhomogeneous shear deformation field.
\end{abstract}

Introduction. - Due to the relevance for cell mechanics, the elastic response of biopolymeric networks has been extensively studied experimentally [1-3]. The cortical cytoskeleton consists of randomly oriented actin filaments that are crosslinked via actin-binding-proteins [4-6]. Cytoskeletal elasticity exhibits unusual features [7], such as stress stiffening [8-11] and negative normal stress $[12,13]$. Particular attention has been paid to the frequency-dependent linear viscoelasticity [14-17], which exhibits a viscoelastic plateau at low and an $\omega^{1 / 2}$ power law at intermediate frequencies for actin networks that are entangled [18-25] or crosslinked by $\alpha$-actinin [26], filamin [27-29], fascin [30], scruin [9] or heavy meromyosin [31,32]. Scaling laws governing the frequency-dependent linear viscoelasticity $G(\omega)$ of polymeric networks have been worked out $[2,33-39]$, but a procedure that allows to quantitatively predict $G(\omega)$ based on single polymer properties and network topology is not available in literature.

We present a bottom-up theory for the linear frequencydependent viscoelastic response of a polymeric network that is based on a systematic and iterative coarse-graining procedure, similar in spirit to the real-space renormalization group approach [40] and to a previous calculation of the static elastic response of self-similar polymer networks [41]. Our method allows for analytic asymptotic analysis and yields at low numerical cost results that are virtually exact when compared with full numeric calculations that are only feasible for small system sizes [42]. As the only input our approach requires the frequencydependent response functions of a single worm-like chain (WLC), which we obtain from the anisotropic mean-field approximation, previously shown to very accurately reproduce simulations and experimental results for the endto-end dynamics of DNA [43]. Alternatively, these input response functions could be taken from simulations or experiments. We show results for square, hexagonal and cubic network connectivities, other connectivities are possible and will be considered in future work. Using cubic lattices and accounting for random network orientation, we find quantitative agreement with experimental data for the linear shear modulus $G(\omega)$ for crosslinked actin solution over a wide frequency range and in particular reproduce the low-frequency viscoelastic plateau and the intermediate-frequency $\omega^{1 / 2}$ scaling. We have thus introduced an efficient bottom-up method that allows to calculate the macroscopic viscoelasticity of networks solely based on viscoelastic properties of the constituent polymer segments.

Method. - We explain our coarse-graining method for the vertical stretching of a $2 \mathrm{D}$ square network in fig. 1 (which in fact dominates the response of a sheared ran- 


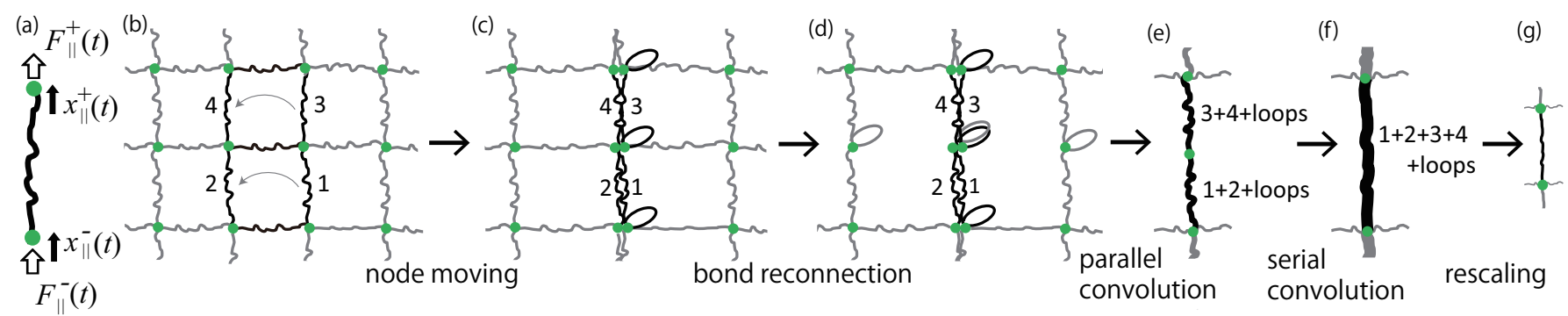

Fig. 1: (a) Setup for the definition of the parallel response functions of a single polymer with time-dependent parallel end-point positions $x_{\|}^{+}(t)$ and $x_{\|}^{-}(t)$ and parallel external forces $F_{\|}^{+}(t)$ and $F_{\|}^{-}(t)$. (b)-(g) Schematic illustration of the five consecutive coarse-graining steps.

domly oriented network, as will be argued below). The complex force response functions $g_{\alpha \beta}^{A B}(\omega)$ of an isolated single polymeric segment that is subject to a finite stretching are defined through the linear-response relation [44] $F_{\alpha}^{A}=\sum_{B=+,-} \sum_{\beta=\|, \perp} g_{\alpha \beta}^{A B}(\omega) x_{\beta}^{B}$, which relates the external force amplitude $F_{\alpha}^{A}$ acting on the two polymer ends to the displacement amplitudes $x_{\beta}^{B}$ of the polymer end points. The indices $A, B=+,-$ refer to the two polymer ends and $\alpha, \beta=\|, \perp$ denote parallel and perpendicular directions with respect to the mean polymer end-toend direction, see fig. 1(a). For symmetric polymers with identical ends, the response is entirely described by the self-response function $g_{\alpha \beta}^{\text {self }} \equiv g_{\alpha \beta}^{++}=g_{\alpha \beta}^{--}$and the crossresponse function $g_{\alpha \beta}^{\text {cross }} \equiv g_{\alpha \beta}^{+-}=g_{\alpha \beta}^{-+}[44]$. Since parallel and perpendicular directions decouple [44], we are left with the four frequency-dependent response functions, $g_{\perp \perp}^{\text {self }}(\omega)$, $g_{\|\|}^{\text {self }}(\omega), g_{\perp \perp}^{\text {cross }}(\omega), g_{\|\|}^{\text {cross }}(\omega)$. The first step in our coarsegraining procedure is a node moving step, where we move every second vertical column of nodes onto the left neighbor column, see fig. 1(b) to (c). This turns half of the horizontal segments into loops as indicated in fig. 1(c). In the bond-reconnection step from fig. 1(c) to (d), in every second row the end points of horizontal segments are reconnected so that they form loops. Note that this does not change the network response since a vertical stretching force does not put horizontal segments under tension. In the third step from fig. 1(d) to (e), we calculate the vertical response functions by convolution of the two parallel and looped segments which amounts to adding the moduli. This step is exact. In the fourth step from fig. $1(\mathrm{e})$ to (f), we eliminate the inner node by convolution of the two segments that are connected in series. Also this serial convolution step is exact on the linear response level $[42,44]$. In the final rescaling step from fig. $1(\mathrm{f})$ to $(\mathrm{g})$, the stretching response is multiplied by a factor of $1 / 2$, so that it corresponds to the response per chain and the limit $n \rightarrow \infty$ exists. After $n$ iterations the viscoelastic stretching response of a network of $2^{n} \times 2^{n}$ nodes is obtained. Similarly, the viscoelastic stretching as well as shear response of $2 \mathrm{D}$ hexagonal and $3 \mathrm{D}$ cubic lattices is obtained (see Supplementary Information (SI) for details [45]).
Results. - In fig. 2(a) we show the real and imaginary parts of the stretching response $g_{\mathrm{st}}(\omega)$ of a $2 \mathrm{D}$ square lattice sketched in fig. 2(b), which results from the self and cross functions as $g_{\mathrm{st}}(\omega)=\left(g_{\|\|}^{\mathrm{self}}(\omega)-g_{\|\|}^{\text {cross }}(\omega)\right) / 2$ $[42,44]$. As input for the complex single-segment response functions in the first iteration step, we use the anisotropic mean-field expressions for a WLC, which have previously been successfully compared with simulations and experiments [43] (see SI [45] for a brief description). The response functions neglect hydrodynamic interaction effects which will be later on accounted for by slender body hydrodynamic theory. We choose a segment contour length $L_{s}=0.1 \ell_{p}$, much smaller than the WLC persistence length $\ell_{p}$, and a fairly large stretching parameter $\chi \equiv 4\left(\ell_{p} f_{0} / k_{B} T\right)^{1 / 2}=10$. Here $f_{0}$ is a stretching force that acts on all segments and reflects the effect of osmotic pressure due to counterions and hydration forces in experimental systems. Note that at the nodal connections we do not account for correlations between tangents of adjoining segments. In the SI [45] we also show results for finite torque coupling at the nodes, which influences the shear response slightly but is irrelevant for the stretch response on the linear-response level. We rescale $g_{\mathrm{st}}(\omega)$ by the effective spring constant $k_{\|} \equiv 4 \ell_{p}^{1 / 2} f_{0}^{3 / 2} /\left(\left(k_{B} T\right)^{1 / 2} L_{s}\right)=$ $k_{B} T \chi^{3} /\left(16 \ell_{p} L_{s}\right)$ and the frequency by the characteristic time $\tau_{0} \equiv \zeta L_{s} / k_{\|}=16 \ell_{p} L_{s}^{2} \zeta /\left(k_{B} T \chi^{3}\right)$, where $\zeta$ is the friction coefficient per length. We present results for up to $n=10$ iterations, corresponding to a system size of $L=2^{n} L_{s}$. At high frequency, we obtain $g_{\mathrm{st}}(\omega) \propto \omega^{3 / 4}$, irrespective of the number of iterations, as expected for WLCs in the absence of hydrodynamic interactions [33]. Both the storage and the loss functions $g_{\mathrm{st}}^{\prime}(\omega)$ and $g_{\mathrm{st}}^{\prime \prime}(\omega)$ develop with rising $n$ an $\omega^{1 / 2}$ powerlaw at intermediate frequencies. For low frequency, the real part $g_{\mathrm{st}}^{\prime}(\omega)=g_{\mathrm{st}}^{\text {plat }} \sim k_{\|} / 2^{n}$ is constant while the imaginary part $g_{\mathrm{st}}^{\prime \prime}(\omega)$ is linear in $\omega$, the expected viscoelastic plateau regime $[30,34]$, both parts depend on the number of coarse-graining steps $n$. As $n$ and thus the system size increases, the crossover frequency $\omega^{*}$ between the plateau and the $\omega^{1 / 2}$ regimes moves to lower frequencies according 

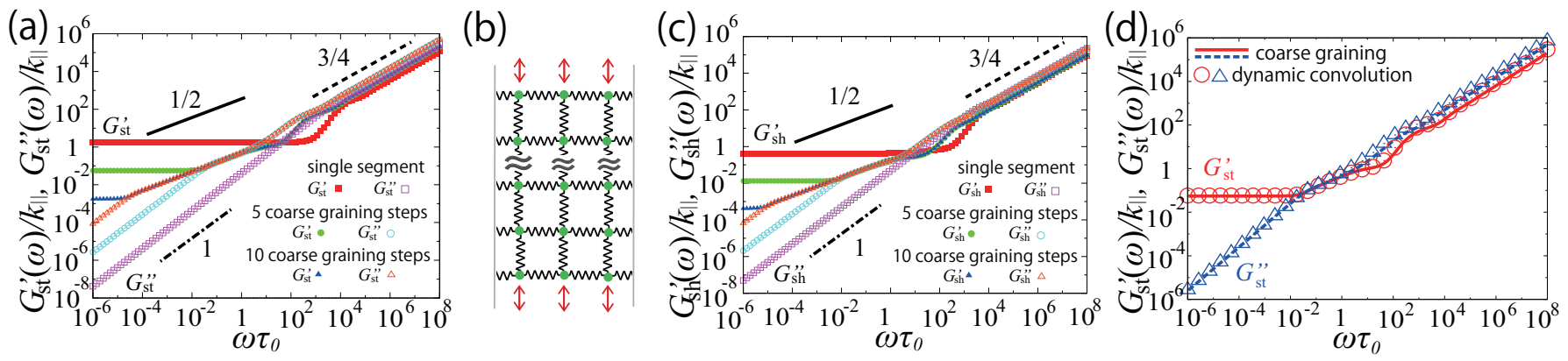

Fig. 2: (a) Coarse-graining results for the complex frequency-dependent linear stretch response $g_{\mathrm{st}}(\omega)$ of a $2 \mathrm{D}$ square lattice network under vertical stretching force after $n=0$ (corresponding to a single segment), 5 and 10 iterations. The segments are worm-like chains with contour length $L_{s}=0.1 \ell_{p}$ and the stretching parameter is $\chi=10$. The straight lines denote power laws as indicated. (b) Network structure used for (a) and (d). (c) Coarse-graining results for the shear response $g_{\mathrm{sh}}(\omega)$ of a hexagonal lattice for $n=0,5$ and 10 iterations. (d) Comparison of the real and imaginary parts of $g_{\mathrm{st}}(\omega)$ for a $2 \mathrm{D}$ square system of size $L=2^{5} L_{s}$ from the present coarse graining method (lines) with numerically exact dynamic convolution results (symbols).

to

$$
\omega^{*} \sim \frac{8 \pi^{2} k_{\|} L_{s}}{\zeta L^{2}}
$$

as will be discussed further below. For a $2 \mathrm{D}$ hexagonal lattice, the shear response $g_{\mathrm{sh}}(\omega)$ in fig. 2(c) shows qualitatively similar behavior.

To demonstrate the accuracy of our coarse-graining method, we in fig. 2(d) compare with numerically exact calculations of the stretching response using the full dynamic convolution theory [42]. Since this method requires inversion of the response matrix, we are in this comparison restricted to relatively small systems with a size of $L=2^{5} L_{s}$, which corresponds to only 5 coarse-graining iterations. Lines denote the real and imaginary parts of $g_{\text {st }}(\omega)$ from coarse-graining, while symbols denote exact dynamic convolution results. The agreement is very good, meaning that the approximate treatment of the boundary conditions in our coarse-graining procedure is accurate (see [45] for details). Note that our coarse-graining method comes at low numerical cost and thus allows to calculate the viscoelastic response for realistic experimental network sizes $L$.

We next investigate how various system parameters influence the results. In fig. 3(a) we compare the stretching response $g_{\text {st }}(\omega)$ of a single WLC with contour length $L=6.4 \ell_{p}$ with that of a $1 \mathrm{D}$ sequence of $2^{5}$ connected WLC segments of the same total contour length and observe slight but significant deviations: While the 1D sequence of connected WLC segments exhibits an extended pure $\omega^{1 / 2}$ regime, as seen in the inset where we plot the logarithmic derivative of $g_{\mathrm{st}}^{\prime \prime}(\omega)$, the single WLC shows a broad crossover of the exponent from $1 / 2$ to $3 / 4$ with increasing frequency. We conclude that the angular decorrelation of connected segments at nodes is essential for the resultant $\omega^{1 / 2}$ scaling in the 2D network response seen in fig. 2(a). In fig. 3(b) we compare results for $1 \mathrm{D}$ sequences with $2 \mathrm{D}$ square and $3 \mathrm{D}$ cubic networks. We find the differences are rather small and also the $\omega^{1 / 2}$ scaling is not modified. From this we conclude that horizontal segments and the network connectivity contribute only weakly to the vertical stretching response. In fig. 3(c) we compare 2D square network moduli for three different parameter combinations of segment length $L_{s}$ and stretching parameter $\chi$. We see that the intermediate-frequency $\omega^{1 / 2}$ scaling is obtained for all three parameter combinations.

Comparison with experimental data. - To quantitatively compare our coarse-graining results with experimental data, we choose a 3D cubic lattice and take care of three additional effects: i) We have so far defined the stretching force response with respect to the end-to-end displacement, whereas in experiments, it is defined with respect to the dimensionless strain, i.e. with respect to the end-to-end displacement divided by the system size $L$. This adds a factor of $L$ in the resulting response function. ii) We calculate the response per polymer segment, whereas in experiments, the response is normalized by the area, which defines the modulus $G$. We take care of this by multiplying the single-chain response by a factor of $1 / \xi^{2}$, the inverse squared mesh size $\xi$, which can be directly obtained from the experimental polymer density [26]. iii) Our calculations consider a perfectly aligned cubic network lattice, whereas in experiments, a polymeric network consists of finite-size domains that exhibit random orientations. This we account for by a correction factor $\phi$, which can be derived from the relation $\sigma_{i j} \sim n_{j}\left[n_{i} n_{k} G_{\text {st }}(\omega)+\left(\delta_{i k}-n_{i} n_{k}\right) G_{\text {sh }}(\omega)\right] n_{l} u_{k l}$ between stress $\sigma_{i j}$ and strain $u_{k l}$ involving the unit vector $n_{i}$ characterizing the local network orientation [33]. In a typical shearing experiment one therefore simultaneously probes shear and stretch moduli $G_{\mathrm{sh}}(\omega)$ and $G_{\mathrm{st}}(\omega)$, according to the network local random orientation. The stretch modulus of a cubic network is much larger than the shear modulus, since shear probes a floppy (i.e. soft) mode where polymer segments are not extended (see fig. S2 in the SI [45]). We thus compare experimental data with only the first term, the stretch modulus. For random orientation one obtains $\left\langle n_{i} n_{j} n_{k} n_{l}\right\rangle=(1 / 15)\left(\delta_{i j} \delta_{k l}+\delta_{i k} \delta_{j l}+\delta_{i l} \delta_{j k}\right)$ 

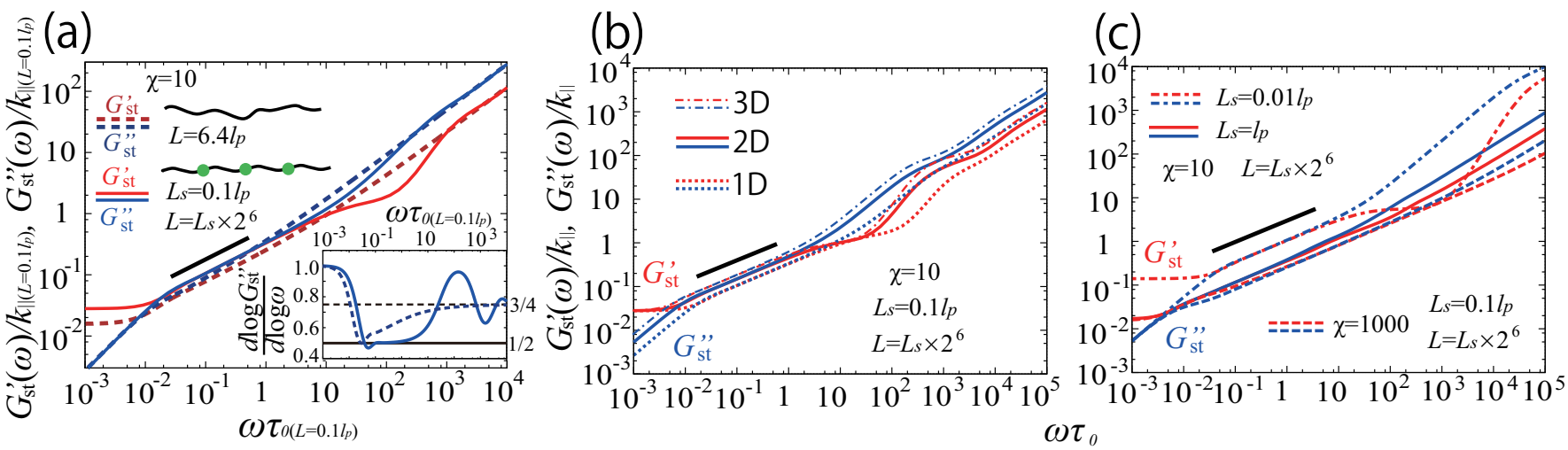

Fig. 3: Red curves denote the storage stretching response $g_{\mathrm{st}}^{\prime}$ and blue curves the loss stretching response $g_{\mathrm{st}}^{\prime \prime}$. The solid bar denotes the $\omega^{1 / 2}$ power law. (a) Comparison of a single worm-like chain (WLC, broken lines) and a 1D sequence of 64 angularly uncorrelated connected WLCs (solid lines) of the same total contour length. As shown in the inset, the power-law exponent for the $1 \mathrm{D}$ sequence is $1 / 2$ over a wide intermediate frequency window, whereas for the single WLC the exponent continuously shifts from $1 / 2$ to $3 / 4$. (b) Comparison of $1 \mathrm{D}$ sequences (dotted lines), 2D square (solid lines) and 3D cubic networks (dashed-dotted lines) of WLCs with $L_{s}=0.1 \ell_{p}$ and $\chi=10$. (c) Comparison of the stretching response of $2 \mathrm{D}$ networks for three different parameter combinations for $\chi$ and $\ell_{p}$, all for the same system size $L$.

[33], giving a correction factor $\phi=1 / 15$. Considering all these effects, we define the modulus to be compared with experimental data as

$$
G(\omega)=\phi L g_{\mathrm{st}}(\omega) / \xi^{2} .
$$

For our comparison in fig. 4, we have to extract the segment contour length $L_{s}$ from experimental conditions. Correlations between polymers in a network are not only due to crosslinkers but also due to entanglements $[46,47]$. Hence, we assume that $L_{s}$ is identical to the entanglement length, which for WLCs is related to the mesh size $\xi$ via $L_{s} \simeq \xi^{4 / 5} \ell_{p}^{1 / 5}$ and involves the persistence length $\ell_{p}$ $[34,35,48]$. Based on the experimentally used actin densities we estimate for the mesh size $\xi=0.47 \mu \mathrm{m}$. Here, $\xi$ is calculated via $\xi \sim 0.3\left(c_{g a}^{\prime}\right)^{-1 / 2}$ with the actin mass density $c_{g a}^{\prime}$ given in units of $\mathrm{mg} / \mathrm{ml}$ and $\xi$ given in units of $\mu \mathrm{m}[23,48]$, and the experimental G-actin concentration of $c_{g a} \sim 9.5 \mu \mathrm{M}[30]$ corresponds to $c_{g a}^{\prime} \sim 0.40 \mathrm{mg} / \mathrm{ml}$. From the persistence length $\ell_{p}=16 \mu \mathrm{m}$ we thus deduce $L_{s}=1.0 \mu \mathrm{m}$. For the system size we adopt $L=2^{7} L_{s}$, which is close to the typical plate-plate separation of experimentally employed rheometers, $L \sim 150 \mu \mathrm{m}[27,30]$. The stretching parameter $\chi$ is an important parameter as it sensitively determines the WLC response functions that are used in the first iteration step [43]. By a fit of the static storage modulus $G^{\prime}(\omega \rightarrow 0 \mathrm{~Hz})$ to the experimental value, $G^{\prime}(\omega \rightarrow 0 \mathrm{~Hz}) \simeq 0.1 \mathrm{~Pa}$, we deduce a value of $\chi=26$. This value corresponds to the ideal gas osmotic pressure for a concentration of $2 \times 10^{-8} \mathrm{M}$, much smaller than the expected concentration of $3.8 \times 10^{-5} \mathrm{M}$ of uncondensed counterions (four per G-actin monomer) in the experimental system [45]. This may indicate the presence of counterion binding effects, attractive interactions between actin filaments or effects due to added salt. The broken lines in fig. 4 indicate the result of our theory without hydrodynamic effects. In this case, the friction coefficient that enters the calculation of the WLC response functions is given by $\zeta=1 /\left(a \mu_{0}\right)$, where $\mu_{0}=1 /(6 \pi \eta a)$ denotes the Stokes mobility of a sphere of radius $a$ for solvent viscosity $\eta$. We use the viscosity of water at $25^{\circ} \mathrm{C}$ as $\eta \sim 0.89 \mathrm{mPa} \cdot \mathrm{s}$ and an actin radius of $a \sim 4 \mathrm{~nm}[2,48,49]$, so that the Stokes mobility comes out as $\mu_{0} \sim 15 \mu \mathrm{m} /(\mathrm{ms} \cdot \mathrm{pN})$. The broken curves in fig. 4 are shifted to lower frequencies compared to experiments. This shift can be accounted for by hydrodynamic effects. Since the hydrodynamic screening length is comparable to the mesh size $\xi$ [50], we can apply slender-body theory using an effective hydrodynamic length of $\xi$. The parallel and perpendicular friction coefficients are thus given by $\zeta_{\|}=(2 \pi \eta) / \ln (\xi /(2 a))$ and $\zeta_{\perp}=2 \zeta_{\|}[34,43]$. The theoretical results with these modified slender-body friction coefficients are shown by solid lines in fig. 4 and nicely agree with the experimental data without further fitting parameters.

Heavy meromyosin (HMM) in the rigor state is a crosslinker protein that is known to form isotropic crosslinked networks for a broad range of crosslinker concentrations $[31,32]$. A more critical check on the assumptions in our theoretical model will be possible by comparison with rigor-HMM network data for varying crosslinker to actin concentration ratios. For example, our assumption of angular chain decorrelation at nodal connections may not be applicable at high crosslinker concentration, where each actin filament will have more than two nodal connections.

We note that the theoretical results are less sensitive to the segment length $L_{s}$ than to the mesh size $\xi$, as shown in fig. S3 in the SI [45]. In fact, the static elastic modulus changes from $G^{\prime}(\omega=0) \simeq 0.10 \mathrm{~Pa}$ for $L_{s}=1.0 \mu \mathrm{m}$ to $G^{\prime}(\omega=0) \simeq 0.15 \mathrm{~Pa}$ when $L_{s}$ is decreased 


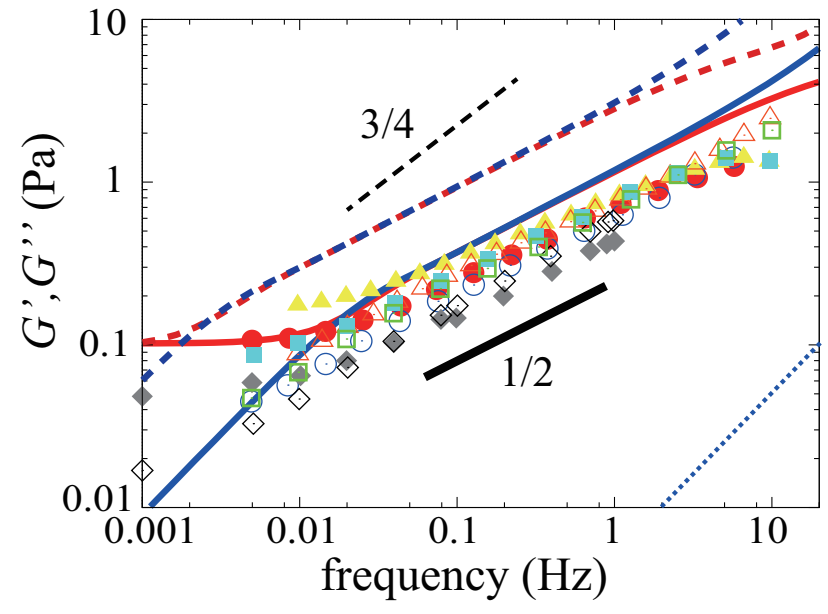

Fig. 4: Comparison of experimental data (symbols) with theoretical results for the complex shear moduli of actin gels. Experimental data without crosslinkers (circles) [25], with added depleting agents (diamonds) [24], with filamin (squares) [29] and fascin (triangles) [30] are shown (molar concentration ratios $R$ of crosslinkers and actin are $R=0.0005$ and $R=0.005$ for filamin and fascin, respectively, and thus low enough so that actin bundling can be neglected). In all experiments, the actin monomer concentration is $9.5 \mu \mathrm{M}$, and the average actin filament length is adjusted to $21 \mu \mathrm{m}$. Filled symbols indicate the storage modulus $G^{\prime}$ and open symbols the loss modulus $G^{\prime \prime}$. Broken and solid lines represent coarse-graining results for 3D cubic lattices without and with hydrodynamics effects, respectively. Red and blue curves show the storage and loss moduli, $G^{\prime}$ and $G^{\prime \prime}$, respectively. In the calculations we use a mesh size $\xi=0.47 \mu \mathrm{m}$, segment contour length $L_{s}=1.0 \mu \mathrm{m}$, and a stretching force parameter $\chi=26$ determined by a fit to the experimental static modulus $G^{\prime}(\omega=0) \sim 0.1 \mathrm{~Pa}$. The $\omega^{1 / 2}$ and $\omega^{3 / 4}$ power laws are denoted by solid and broken straight lines, respectively. The blue dotted line indicates the loss modulus $G^{\prime \prime}=\omega \eta$ for pure water at $25^{\circ} \mathrm{C}$ with a viscosity $\eta=0.890 \mathrm{mPa} \cdot \mathrm{s}$, which is much smaller than the polymer network response.

to $L_{s}=\xi=0.47 \mu \mathrm{m}$. Since $L_{s}$ is inversely proportional to the crosslinker concentration, this trend is consistent with experimental observations [24, 29,51].

Discussion. - The $\omega^{1 / 2}$ power law, which in the experimental data is present over almost three orders of magnitude in frequency, see fig. 4, arises in our model due to the inhomogeneous propagation of tension into the network and involves angular chain decorrelations at nodal connections, as demonstrated in fig. 3(a). It is thus slightly different from the tension-relaxation mechanism operative for a sufficiently long single chain if $L \gg \ell_{p}[34,52]$. Interestingly, a pronounced intermediatefrequency regime with $\omega^{1 / 2}$ also results for a network of Gaussian chains $[22,23,53,54]$, which we derive analytically in the SI [45], and thus is not restricted to WLC networks.

Note that a $\omega^{1 / 2}$ power-law has also been predicted in the low-frequency regime for reversible crosslinkers
[36]; this however does not explain the universality of the power-law scaling, which in the experimental data is present over almost three orders of magnitude in frequency and is independent of the cross linker concentration [30] (fig. 4).

Our results imply that while the shear modulus is independent of system size in the plateau regime, it exhibits a linear dependence on the system size in the powerlaw regime at intermediate frequencies, which is caused by a finite and frequency-dependent penetration depth of the shear deformation field into the polymeric network. Such effects are well-known for simple viscous fluids, where the frequency-dependent shear penetration depth scales as $\sqrt{\eta /(\omega \rho)}$ and only depends (besides frequency) on the fluid density $\rho$ and fluid viscosity $\eta$ [55]. This implies that even for pure water, the imaginary shear modulus measured in a rheometer with plate separation $L$ becomes $L$-dependent at frequencies larger than $\omega^{*}=\eta /\left(\rho L^{2}\right)$ and acquires a powerlaw different from the low-frequency behavior $G^{\prime \prime}=\omega \eta[55]$. For pure water and for a typical plate separation of $L=100 \mu m$ the crossover frequency is about $\omega^{*}=100 s^{-1}$ and thus slightly above the range considered in fig. 4.

The spatially inhomogeneous tension propagation in the powerlaw regime is reflected by the dependency of the crossover frequency $\omega^{*}$ between the plateau and $\omega^{1 / 2}$ regimes in eq. (1) on the system size $L$. To substantiate this, we estimate $\omega^{*}$ based on simple scaling ideas: The longest relaxation time of a single WLC segment in the longitudinal direction is, using the weak-bending approximation valid for large stretching parameter $\chi \gg 1$, given by $\tau_{l} \sim \chi^{-1} \zeta /\left(k_{B} T \ell_{p}\left(2 \pi / L_{s}\right)^{4}\right)$. Since tension propagation can be described as a diffusion process with diffusion constant $D=L_{s}^{2} / \tau_{l}$ as long as the angles of the connected segments are decorrelated, the length scale over which tension propagates within time $t$ is given by $\sim L_{s}\left(t / \tau_{l}\right)^{1 / 2}$. Associating the length scale with the system size $L$, the crossover frequency is obtained as $\omega^{*} \sim D / L^{2}$, which is equivalent to eq. (1) (see the SI for an explicit derivation [45]).

The neglect of hydrodynamic interactions for the calculation of the input single-chain response functions deserves some discussion. The effect of hydrodynamic interaction on the WLC response functions has been previously estimated [43] and can rather accurately be accounted for by a shift of time scales according to the hydrodynamic slender-body approximation, as we have done for the comparison in fig. 4. In the calculation of the single chain response functions, the background solvent is assumed to rest relative to the chain. When constructing the network response function, this means that we implicitly assume that the background solvent moves together with the polymer network. This is unproblematic in the low-frequency plateau regime, where the shear deformation field is homogeneous. However, in the high-frequency power-law regime the polymeric shear deformation field becomes inhomogeneous and the question arises whether 
there is relative motion between the polymer network and the background fluid (which would not be accounted for in our model and which would have to be treated by a much more involved two-fluid model [56]). In fig. 4 we show the viscous shear response of pure water (broken blue line) which is given by $G^{\prime \prime}=\omega \eta$. For a frequency around $\omega=0.1 s^{-1}$ the pure water response is smaller than the polymeric network response by two orders of magnitude, meaning that the viscoelastic response is dominated by the polymeric network. We thus conclude that the water shear deformation field will follow the polymeric network, in agreement with our model assumptions. Clearly, the incompressible background fluid cannot follow the network when it is stretched in a non volume-conserving fashion, so presumably there is some residual relative motion between the fluid and the polymer network, which is not accounted for in our model. Thus it would be desirable to resolve the relative motion of the background fluid and the polymer network under shear in more detail in future ramifications of the present model.

The system size dependence of the shear modulus in the intermediate powerlaw regime should be detectable experimentally. Surprisingly, only very few experimental studies investigated the system-size dependence of the viscoelastic response of biopolymer gels $[57,58]$, and no system-size dependent measurement of the frequency-dependent linear viscoelasticity of an actin gel in the intermediate frequency regime exists. Due to the relevance of the viscoelasticity of gels in the microscale confinement of cells and organelles [59], systematic experimental work in that direction would be highly desirable.

The good agreement with experimental data in fig. 4 demonstrates that our method correctly predicts the macroscopic viscoelastic response of WLC networks based solely on the viscoelastic single-chain response functions. In this comparison, the osmotic solution pressure, which is fitted to the low-frequency experimental plateau modulus and which determines the single-chain response functions, is shown to be a very important experimental parameter. It would be interesting to systematically vary this parameter in future experiments. In the comparison with the experimental data we used an ordered cubic network connectivity. It will be interesting to develop similar coarsegraining methods for random networks where the local network connectivity can be characterized by distribution functions [60].

\section{$* * *$}

We thank W. K. Kim and M. Kanduč for discussions. We acknowledge support by the Alexander von Humboldt Foundation, the JSPS Core-to-core Program and the DFG via grant GRK 1558.

\section{REFERENCES}

[1] Sackmann E., Macromol. Chem. Phys., 195 (1994) 7.
[2] Mofrad M. R. K., Annu. Rev. Fluid. Mech., 41 (2009) 433.

[3] Lieleg O. and Bausch A. R., Soft Matter, 6 (2010) 218.

[4] Bray D., Heath J. and Moss D., J. Cell Biol., 4 (1986) 4.

[5] Rivero F., Köppel B., Peracino B., Bozzaro B., Siegert F., Weijer C. J., Schleicher M., Albrecht R. and Noegel A. A., J. Cell Sci., 109 (1996) 2679.

[6] Bausch A. R. and Kroy K., Nature Phys., 2 (2006) 231.

[7] MacKintosh F. C., Käs J. and Janmay P. A., Phys. Rev. Lett., 75 (1995) 4425.

[8] Gardel M. L., Shin J. H., MacKintosh F. C., Mahadevan L., Matsudaira P. A. and Weitz D. A., Science, 304 (2004) 1301.

[9] Gardel M. L., Shin J. H., MacKintosh F. C., Mahadevan L., Matsudaira P. A. and Weitz D. A., Phys Rev. Lett., 93 (2004) 188102.

[10] Storm C., Pastore J. J., MacKintosh F. C., LubenSKy T. C. and JANMEY P. A., Nature, 435 (2005) 191.

[11] Gardel M. L., Nakamura F., Hartwig J. H., Crocker J. C., Stossel T. P. and Weitz D. A., Proc. Natl. Acad. Sci., 103 (2006) 1762.

[12] Janmay P. A., McCormick M. E., Rammensee S., Leight J. L., Georges P. C. and MacKintosh F. C., Nature Mat., 6 (2006) 48.

[13] Conti E. and MacKintosh F. C., Phys. Rev. Lett., 102 (2009) 088102.

[14] Jen C. J. and Mcintire L. V. and Bryan J., Arch. Biochem. Biophys., 216 (1982) 126.

[15] Sato M., Scwarz W. H. and Pollard T. D., Nature, 325 (1987) 828.

[16] Elson E. L., Ann. Rev. Biophys. Biophys. Chem., 17 (1988) 397.

[17] Janmay P. A., Hvidt S., Lamb J. and Stossel T. P., Nature, 345 (1990) 89.

[18] Ruddies R., Goldmann W. H., Isenberg G. and SaCKMANn E., Euro. Biophys. J., 22 (1993) 309.

[19] Ziemann F., RÄDler J. and Sackmann E., Biophysical Journal, 66 (1994) 2210.

[20] Crocker J. C., Valentine M. T., Weeks E. R., Gisler T., Kaplan P. D., Yodh A. G. and Weitz D. A., Phys. Rev. Lett., 85 (2000) 888.

[21] Gardel M. L., Valentine M. T., Crocker J. C., Bausch A. R. and Weitz D. A., Phys. Rev. Lett., 91 (2003) 158302.

[22] Uhde J., Feneberg W., Ter-Oganessian N., Sackmann E. and Boulbitch A., Phys. Rev. Lett., 94 (2005) 198102 .

[23] Liu J., Gardel M. L., Kroy K., Frey E., Hoffman B. D., Crocker J. C., Bausch A. R. and Weitz D. A., Phys. Rev. Lett., 96 (2006) 118104.

[24] Tharmann R., Claessens M. M. A. E. and Bausch A. R., Biophysical Journal, 90 (2006) 2622.

[25] Semmrich C., Larson R. J. and Bausch A. R., Soft Matter, 4 (2008) 1675.

[26] Müller O., Gaub H. E., Bärmann M. and Sackmann E., Macromolecules, 24 (1911) 3111.

[27] Gardel M. L., Nakamura F., Hartwig J., Crocker J. C., Stossel T. P. and Weitz D. A., Phys. Rev. Lett., 96 (2006) 088102.

[28] Wagner B., Tharmann R., Haase I., Fischer M. and Bausch A. R., Proc. Natl. Acad. Sci., 103 (2006) 13974. 
[29] Schmoller K. M., Lieleg O., and Bausch A. R., Biophysical Journal, 97 (2009) 83.

[30] Lieleg O. and Bausch A. R., Phys. Rev. Lett., 99 (2007) 158105.

[31] Tharmann R., Claessens M. M. A. E. and Bausch A. R., Phys. Rev. Lett. , 98 (2007) 088103.

[32] Luan Y., Lieleg O., Wagner B. and Bausch A. R., Biophysical Journal, 94 (2008) 688.

[33] Gittes F. and MacKintosh F. C., Phys. Rev. E, 58 (1998) R1241.

[34] Morse D. C., Macromolecules, 31 (1998) 7044.

[35] Morse D. C., Macromolecules, 31 (1998) 7030.

[36] Broedersz C. P., Depken M., Yao N. Y., Pollak M. R., Weitz D. A. and Mackintosh F. C., Phys. Rev. Lett., 105 (2010) 238101.

[37] Müller K. W., Bruinsma R. F., Lieleg O., Bausch A. R., Wall W. A. and Levine A. J., Phys. Rev. Lett., 112 (2014) 238102.

[38] Atakhorrami M., Koenderink G. H., Palierne J. F., MacKintosh F. C. and Schmidt C. F., Phys. Rev. Lett., 112 (2014) 088101.

[39] Broedersz C. P. and MacKintosh F. C., Rev. Mod. Phys., 86 (2014) 995.

[40] Kadanoff L. P., Annals. of Phys., 100 (1976) 359.

[41] Jones J. L. and BAll R. C., Macromolecules, 24 (1991) 6369.

[42] von Hansen Y., Rode S., and Netz R. R., Euro. Phys. J. E, 36 (2013) 137.

[43] Hinczewski M. and Netz R. R., Macromolecules, 44 (2011) 6972.

[44] Hinczewsini M., von Hansen Y. and Netz R. R., Proc. Natl. Acad. Sci., 107 (2010) 21493.

[45] See Supplemental Material, for details of the presented method and evaluation of the osmotic pressure.

[46] Dichtl M. A. and Sackmann E. , Proc. Natl. Acad. Sci. 99 (2002) 6533.

[47] Semmrich C. , Storz T. , Glaser J. , Merkel R., Bausch A. R. and Kroy K., Proc. Natl. Acad. Sci., 104 (2007) 20199.

[48] Schmidt C. F., Bärmann M., Isenberg G. and SackMANN E., Macromolecules, 22 (1989) 3638.

[49] Niederman R., Amrein P. C. and Hartwig J., J. Cell Biol., 96 (1983) 1400.

[50] Richter D., Binder K., Ewen B. and Stühn B., J. Phys. Chem., 88 (1984) 6618.

[51] Lieleg O., Claessens M. M. A. E., Heussinger C., Frey E. and Bausch A. R., Phys. Rev. Lett., 99 (2007) 088102 .

[52] Hiraiwa T. and Оhта T., Macromolecules, 42 (2009) 7553.

[53] Gurtovenko A. A., Gotlib Y. Y. and Kilian H.-G., Macromol. Theory Simul., 9 (2000) 388.

[54] Toshchevikov V. P., Gotlib Y. Y. and Blumen A., Macromol. Theory Simul., 16 (2007) 359.

[55] Erbaş A., Podgornik A. and Netz R. R., Euro. Phys. J. E, 32 (2010) 147.

[56] DE Gennes, P.-G., Macromolecules, 9 (1976) 587, Macromolecules, 9 (1976) 594.

[57] Alvarez, M. D., Canet, W. and Fernández, C., J. Food Proc. Eng., 30 (2007) 267.

[58] Arevalo, R. C., Urbach, J. S. and Blair, D. L., Biophys. J., 99 (2010) L65.
[59] Clamessens, M. M. A. E., Tharmann, R., Kroy, K., and Bausch, A. R., Nat. Phys., 2 (2006) 186.

[60] Berker A. N. and Kaya H., Phys. Rev. E, 62 (2000) R1469. 